\title{
Performance evaluation for vehicular ad-hoc networks based routing protocols
}

\author{
Hussain Falih Mahdi', Mohammed Salah Abood ${ }^{2}$, Mustafa Maad Hamdi ${ }^{3}$ \\ ${ }^{1}$ Computer Engineering Department, Faculty of Engineering, University of Diyala, Iraq \\ ${ }^{2}$ School of Information and Electronics, Beijing Institute of Technology, Beijing, China \\ ${ }^{3}$ Department of Computer Engineering Techniques, Al-Maarif University College, Al-Anbar, Iraq
}

\begin{tabular}{l} 
Article Info \\
\hline Article history: \\
Received Sep 27, 2020 \\
Revised Dec 20, 2020 \\
Accepted Jan 22, 2021 \\
\hline
\end{tabular}

Keywords:

MOVE

NS-2.35

Routing protocols

SUMO

VANET

\begin{abstract}
VANET is a branch of MANETS, where each vehicle is a node, and a wireless router will run. The vehicles are similar to each other will interact with a wide range of nodes or vehicles and establish a network. VANETs provide us with the infrastructure to build new solutions for improving safety and comfort for drivers and passengers. There are several routing protocols proposed and evaluated for improving VANET's performance. The simulator is preferred over external experience because it is easy, simple, and inexpensive. In this paper, we choose AODV protocol, DSDV protocol, and DSR protocol with five different nodes density. For each protocol, as regards specific parameters like (throughput, packet delivery ratio, and end- to- end delay). On simulators that allow users to build real-time navigation models of simulations using VANET. Tools (SUMO, MOVE, and NS-2) were used for this paper, then graphs were plotted for evaluation using Trace-graph. The results showed the DSR is much higher than AODV and DSDV, In terms of throughput. While DSDV is the best choice because of the low average end to end delay. From the above, we conclude that each strategy has its own negative and positive aspects that make it ideally suited to a particular scenario than other scenarios.
\end{abstract}

This is an open access article under the CC BY-SA license.

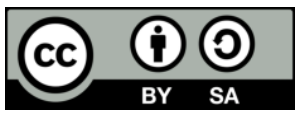

Corresponding Author:

Hussain Falih Mahdi

Computer Engineering Department, Faculty of Engineering

University of Diyala

Baquba, Diyala Province, Iraq

Email: Hussain.mahdi@ieee.org

\section{INTRODUCTION}

Recent advances in communications technologies allow a wide range of different types of networks to deployed in diverse environments to be developed and implemented. I take into consideration an increase in energy efficiency, lower latency, and smooth connectivity [1]. One of these networks that have received a lot of attention in recent years is the vehicular ad-hoc network (VANET) [2-4]. VANET is a part or branch of MANETS built to achieve transportation protection [5], reliability, health, death, and efficiency by integrating with current technologies, each node of a vehicle operating in VANET as considered a vector as seen in Figure 1. Several routing protocols exist, such as dynamic source routing (DSR), ad hoc on-demand distance vector routing (AODV), destination sequence distance vector (DSDV), OLSR, and TORA. Each has a unique action mechanism [6,7]. In this paper, we explored the ability to compare results using SUMO under NS-2 simulator with AODV, DSR, and DSDV protocols based on measurements like (throughput, endto-end delay (E2ED), and packet delivery ratio (PDR)). Controlling and controlling the movement of vehicles in a particular city, especially during emergencies such as congestion at certain times or due to various and 
varied accidents is one of the important challenges facing smart transportation systems [8]. We note that the quality of service of VANET is the basis for performance excellence in smart city systems. VANET primarily depends on the basic routing protocol type if proactive DSDV or reactive AODV and DSR [9, 10].

The limitations of NS-2 are a simulation and it simplifies the structure itself in the real world [11, 12]. Particularly for WLAN simulation, because there are so many parameters that change rapidly within a few periods which are difficult to address or avoid. Processor speed is a concern, too. For e.g, simulating 500 knots with a random path point movement model in a $5 \times 1$ square kilometer area with 100 connections for 600 seconds, which takes several days, may also take as much memory RAM on a $2 \mathrm{GHz}$ computer [13, 14]. The absence of an implementation model makes it inoperative in applications-to-network protocol-interactive settings, it has been developed by many developers and includes several intrinsic bugs which are known and unknown, the NS-2 works well till 100 nodes, and its efficiency decreases as the number of nodes increases. Due to its object-oriented architecture and it does not sit well with WSNs. Finally, it is difficult to use C++ programming and OTCL scripts $[15,16]$.

In this study is to demonstrate the simulation of VANET the methodology to used tools possible, which is possible to be available to simulate VANET using network simulation in NS-2. So, the problem is not just to compare three protocols but to shed light on the simulation process and how VANET works using the tools available in NS-2. Therefore, we are using SUMO because it helps to investigate several research topics like a traffic light, route of algorithm choice, and simulating vehicular communication. Hence the system is used for simulating automated driving or traffic control techniques in various papers. SUMO is not just a traffic simulation, but a series of software and programs that help prepare traffic simulations and execute them.

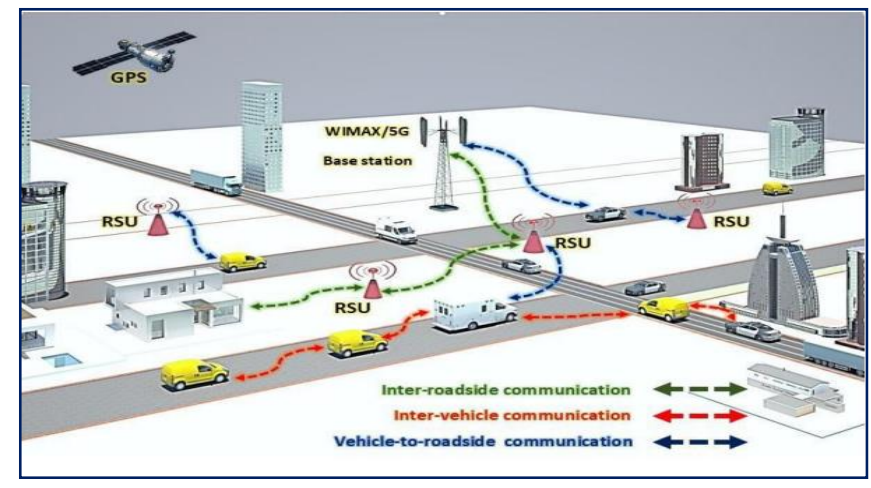

Figure 1. Vehicular ad-hoc network [17]

So, in this paper, we will study wireless networks in a general and detailed study of AODV, DSDV, and DSR protocols in five separate node density scenarios $(10,20,30,40$, and 50) and speed of nodes to try to analyze performance for different parameters. Simulation methods will be used primarily as a generator for the move-urban mobility simulation (SUMO) for implementing the proposed protocol.

a. Study the effect of node density on both constructive and collaborative routing methods for VANET efficiency routing protocols. Using VANET, this study will suggest the most possible protocol and routing methods.

b. The important goal in the paper is to highlight the simulation process and how to work VANET using available tools in NS-2.

c. The results of this work will then be debated, compared, analysis and then the best routing protocol will be given.

The main contributions in this paper, the ability to compare results using SUMO under NS-2 simulator with AODV, DSR, and DSDV protocols based on measurements like throughput, end to end delay, and PDR. We note that the quality of service of VANET is the basis for performance excellence in smart city systems. VANET primarily depends on the basic routing protocol type if proactive DSDV or reactive AODV and DSR. The NS-2 works well till 100 nodes and its efficiency decreases as the number of nodes increases. Therefore, in this paper, a maximum of 50 nodes was used within 5 different scenarios for testing the results by using SUMO because it helps to investigate several research topics like a traffic light, and so on. Finally, the results of this work with the best routing protocol will be given.

In section 2, we'll look at a related work selected for seven preceding papers and briefly showed the specifications of each paper to indicate our vision that we worked on in this paper and a detailed look at

Performance evaluation for vehicular ad-hoc networks based routing protocols (Hussain Falih Mahdi) 
VANET routing protocols like AODV, DSR, and DSDV. A summary of some of the latest search suggestions using SUMO/NS-2. In section 3, there will be a scenario to evaluate the performance of the VANET routing protocols and pick the best protocol for the best highway scenario. In section 4, five highway network scenarios are shown, each with a different number of nodes to measure the impact of traffic load on the transmission and we highlighted the simulation process and how to work VANET using available tools in NS-2. Section 5, running the simulation under an urban mobility simulator and showing the parameters used. In section 6, the final result is drawn to track a specific file to see the drawn result and it can be easily evaluated after discussion because the evaluation focuses on the different performance measures that were used in this paper, and the last part of section 6, we discussed and summarized the results of the research according to the five scenarios, then we studied and analyzed the density factor of the vehicle node affecting the performance of the routing protocols. Finally, in section 6, we concluded that based on theoretical analysis, the findings matched the predicted results. Of the three protocols, it was noted that the DSR efficiency was the best protocol for the transport rate and the packet delivery rate. while DSDV was the highest end-to-end protocol among the protocols.

\section{RELATED WORK}

During the research, we noticed that there are many previous works in the literature, but we will look at the most important ones that have compared and analyzed the performance of routing protocols based on topology. Nonetheless, the researcher and the recipient feel the need for a lot of qualitative comparisons in addition to a lot of performance assessment studies and work that helps assess the performance of these protocols in the VANET environment under NS-2.

The idea of using NS-2 to trying to analyze the performance of with regards various parameters is not new. Especially with the appearance of NS_3, but to highlight the simulation process and how to work VANET using available tools in NS-2. Also, VANETs and MANETs have many similar characteristics, especially during the past years except for the absence of infrastructure in the case of MANET [18]. So in this paper will look at a survey of routing protocols of VANET such as (AODV protocols, DSDV protocols, and DSR protocols) to network and traffic simulators then traffic control interface module [19]. The process of recognizing, managing, and using communication methods is not easy for fast-moving vehicles on VANET because it consists of mobile nodes with a dynamic structure. In the relevant and similar fields of our paper, extensive research has been observed on MANET-guidance protocols compared to VANET, with few and foggy studies on systematic comparison and A performance appraisal study between them (VANETs and MANETs) [20-22].

The researchers in [7] described the determine a strategic hinge gap based on its applicability to a particular application. In other words, it offers a thorough overview of various alignment methods in terms of their benefits and drawbacks and a crucial debate on each classification. The researchers in [23] suggested start the path flow manually from one node to the available parking node indicated in this paper. Then the Network tracing was tested using NS-2 using algorithms such as AODV and DSR. We evaluate the estimation of network monitoring and the results.

The study in [24] shows, provides routing protocol optimization like better link-state routing and dedicated on-demand space. But in the article [25] discusses the latest advances in intermodal traffic approaches, simulator linking and concept creation, and testing on the example of the SUMO open-source traffic simulator.

In article [26] shows that is dependent on the geographical location of the vehicle by making available a modern guidance protocol. Any exploration of these protocols' design drivers and their pros and cons. The researchers have defined several potential directions for the use of this protocol class for future study. In article [27] presented the most commonly encountered contemporary measures in the different hypotheses and conditions for their use. This analysis assists in choosing protocols or generating benchmarks while a new protocol is being developed. This analysis of the multi-metric routing protocols of VANET, then a description of these multi-metric routing protocols is provided.

In article [28] shows a comparison of the results of Three common routing protocols like us (AODV), (DSDV) and (AOMDV) on specific traffic setting. These three protocols have been extensively checked for various parameters, like throughput, PDR average, END2END delay, and energy usage.

Through the above studies, it is clear to us that the researchers focused on comparison more than they adopted the simulation process and how VANET works using the tools available in NS-2, and they did not show the importance of using SUMO which helps in achieving many research topics. Rather, it represents a series of programs that assist in the preparation and implementation of traffic simulations, which will be illustrated in this paper. 


\section{ROUTING PROTOCOLS OF VANET}

It uses connection information within the network to transfer data packets from the source to the destination. We have used DSDV from proactive protocols, then used AODV and DSR from reactive protocols, as shown in Figure 2.

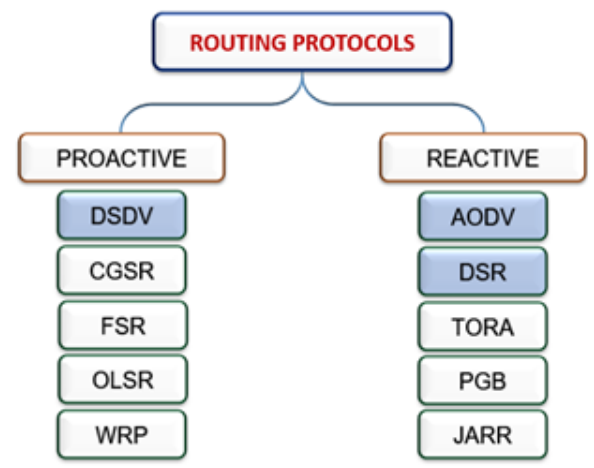

Figure 2. Types of Classification of VANET protocols [6]

\subsection{The DSDV routing protocol}

It is a proactive protocol for routing a unicast broadcast network such as WRP and DSDV and relies on the commonly used Bellman-Ford algorithm. However, its mechanisms are completely different to improve routing performance in dedicated mobile networks. In DSDV routing tables, the next hop to a destination is stored by input, and the cost measure of the route to the destination. DSDV uses serial numbers to distinguish old tracks from new tracks and prevent the creation of track loops. Each node periodically sends updates to its immediate neighbors, including its routing information [2].

\subsection{AODV protocol}

A reactive unicast routing protocol needs to keep active information about route forwarding. Routing information is kept in routing tables in nodes in AODV. The portable node operates the next steer hop, which currently includes paths with a path. The routing table entry expires until it is used or reactivated within the specified expiration period $[2,29]$.

\subsection{The DSR protocol}

It is an interactive unicast routing protocol that uses the algorithm for routing the source. Each data packet inside the source routing algorithm contains complete access and deployment routing information. Additionally, every node in the DSR uses buffering technology to keep the information you have learned about the path. The DSR has two main phases: the road discovery phase and the maintenance phase of the track. The source node that contains the routing information first considers the path cache before sending any package to see if the required path is available or not. The main benefits of DSR are that it is a reactive (ondemand) protocol, ensuring that it does not confuse the network with routing changes because only the path is specified on demand. In intermediate nodes, the information in the cache is used to lower the upper path [21]. Thus, conclude from the above articles, we infer the assessment was AODV demonstrated better efficiency than other protocols with some of the notes.

Table 1. Performance evaluation

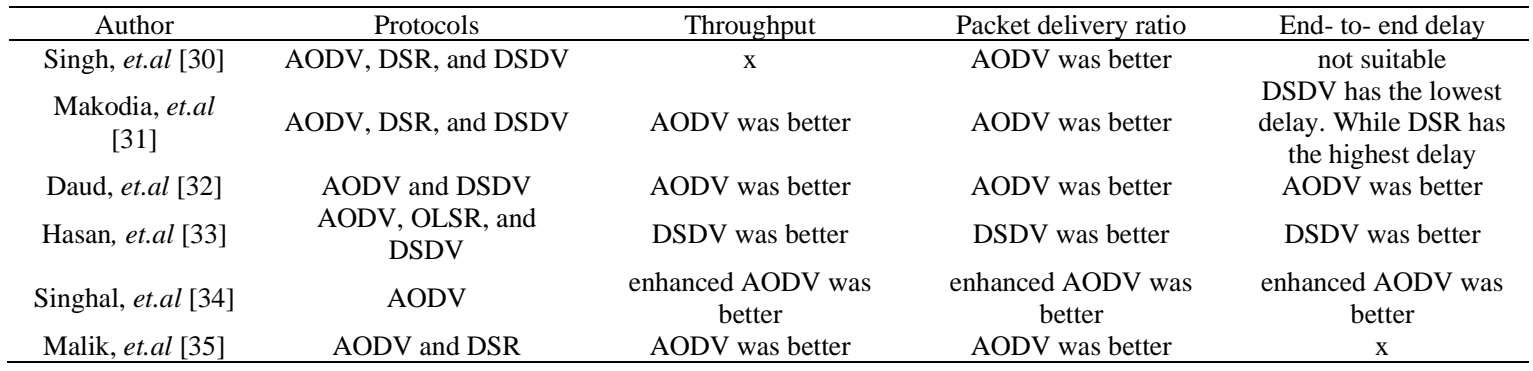




\section{METHODOLOGY}

The objective of the methodology is to show how we conducted this paper stages as shown in Figure 3. This paper aims to assess the effectiveness and selection of the best VANET routing protocols for the highway scenario. The routing protocols that are tested are AODV and DSR (reactive), and one proactive is DSDV. The first step in our methodology is to conduct research on a dedicated network and how it differs from the regular network (infrastructure network) using network simulation by the NS-2 simulator under SUMO.

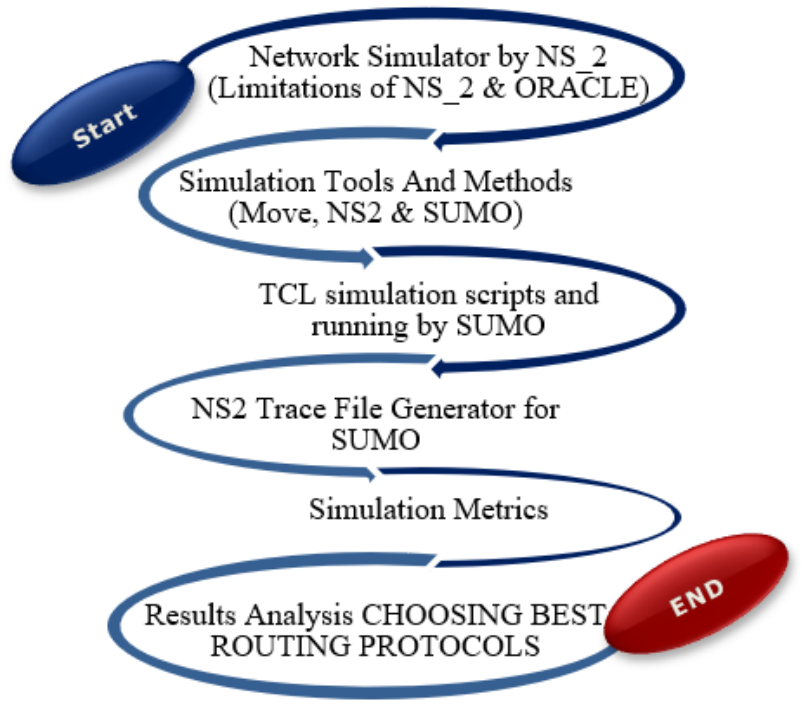

Figure 3. Methodology stages

The second stage moves to understand and conduct research on MANET which is a dedicated wireless network, to know its properties and routing protocols. We will use SUMO to create a TCL file. Why used SUMO? Because it is an open-source and a small and lightweight model for road travel. It helps the user to create custom road topology, as well as import ready-made map formats from many cities and towns around the world. It also supports the application of speed limits and the concept of lane numbers, intersections, lights, and others. SUMO also offers the vehicles the ability to allocate identified users as well as random routes. After finish sets, we can represent all the above with the GUI mode of SUMO.

In the third stage, after understanding MANET, vehicular ad hoc network (VANET) becomes easy to deal with. In the fourth and final stage of our approach, a network simulator called NS-2 is used to test the performance of the proposed routing protocols, the tests are evaluated using different routing methods for specific scenarios, and finally determining the better routing protocol for a specific scenario. In this paper, three protocols are selected to be evaluated which are DSDV, AODV, and DSR. The NS-2 network simulators were used for the highway scenario to verify the VANET simulation. Performance is assessed in terms of throughput, packet delivery rate, END2END Delay, and standardized routing costs. The result is stored in a trace file and shown using the NAM script. in the end, the results are figured using an excel file.

\section{SIMULATION}

\subsection{Network simulator by NS-2}

It is an event-driven, open-source system developed mainly for study in electronic communication networks, it can be summed up:

a. It is a separate event simulation for network research as well as package level work.

b. Simulation language is OTCL and $\mathrm{C}++$, in addition to the GUI property.

c. It provides great emulation support for a range of protocols like UDP, TCP, HTTP, DSR, and FTP.

d. Wireless network simulation. Based on Unix. And NS-2 Supports for multiple protocols.

It supports a lot of algorithms that guide and wait. Routing and broadcasting on a LAN are some examples of routing algorithms, where the circular robin deficit, fair-queue, and FIFO are all queued algorithms supported by NS-2. 


\subsection{Simulation tools and methods}

The simulation framework built using TCL uses two methods to simulate the application and test its performance:

\subsubsection{Move}

Enables users to quickly construct realistic simulation scenarios without complicating the writing of simulation texts and to learn about the simulation's internal features by offering a set of graphical user interfaces that simplify the development of simulation text, and uses the navigator generator tool for the vehicle networks tool to facilitate users to quickly create realistic mobility models for VANET simulations. MOVE is currently being developed in Java and is planned with the SUMO Open-source small traffic simulator also. MOVE output is a navigation tracking file that includes details about practical vehicle motion that can be immediately used with common simulators by NS-2 [36, 37]. MOVE consists of two key components: the first is the car motion editor and the second, as seen in Figure 4, is the map editor. The map editor is used to construct route topology. We are now creating a road map that users can create or manually build. The car travel editor helps the user to determine the movement of the vehicle on a specific trip and the route for each car. MOVE outputs is a navigation effect generated based on the user input shown in the car motion editor and map editor, which can used by a simulator such as NS-2 to simulate realistic car movement [36].

\subsubsection{NS-2 availability, architecture, and languages}

Network simulator (NS-2) is an event-controlled simulator developed at UC Berkeley. We use NS-2 to simulate the protocol. Provides great support for simulating TCP, routing, and multicast protocols over wired and wireless networks. NS-2 code is either written in C++ or OTCL and stored in a separate file executed by the OTCL compiler, generating an output NAM (network animator) script. It then uses the code script to map the nodes at a specific location and shows the output of the nodes that communicate with each other which consists of two simulators. NAM is eventually used to imagine simulations. (SUMO1\&SUMO2 (HTTP)). NS-2 outputs the simulation results after the simulation, on an animation or text basis. It uses tools like NAM and X-Graph graphically and interactively to interpret results [13].

\subsubsection{Simulation of urban mobility}

Certainly, there are many tools available for simulating road traffic microscopy, for example, FARSI and VISSIM but in this study, we will rely on simulation of urban mobility (SUMO) being a common tool for simulating road traffic between the search community [38]. SUMO is an open-source simulator for microscopic and minor road travel. It helps the user to build custom road topology as well as import from several cities and towns around the world different ready-made map formats. The last post helps to create a topology for methods used in the real world. Z. Fu [39] SUMO also supports micro-simulation functions, such as load speed limits, number of lanes, intersections and traffic lights, vehicles with unique features such as vehicle length and idle properties. SUMO also provides the option of assigning the vehicles to both specified users and random routes. Modeling the public transport system also has an option, as each vehicle arrives and departs according to a schedule and all user inputs are in XML format. The created route topology can also be visualized using SUMO's GUI mode. In this Figure 5, explain the total of vehicles and how many vehicles are running and simulation ended time.

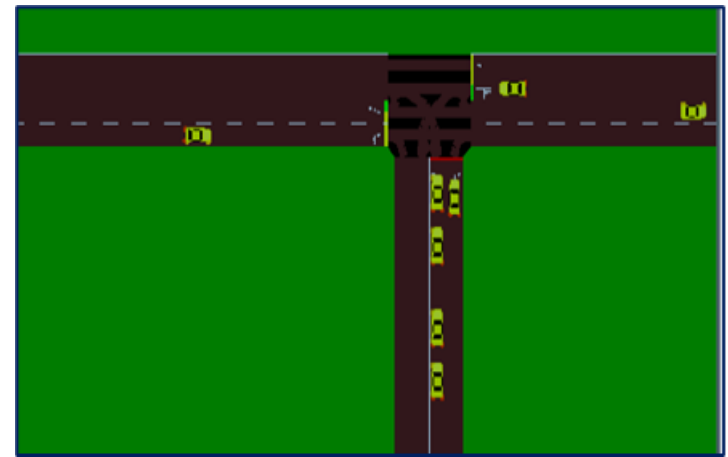

Figure 4. Visualization of vehicle movements

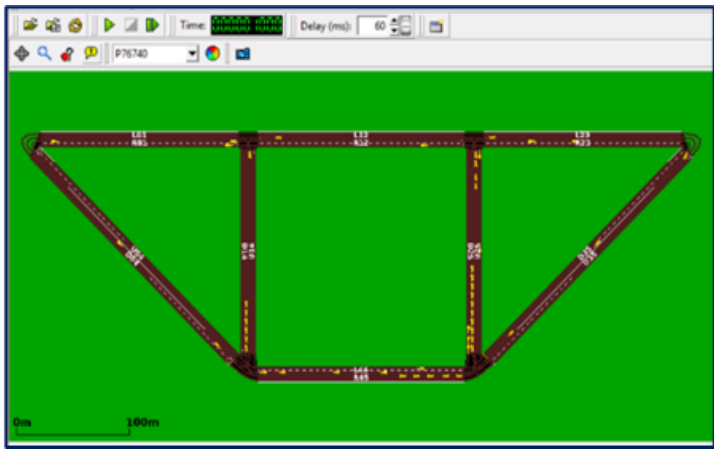

Figure 5. SUMO visualization 


\subsection{Simulation configuration}

To model a network, VANET output is evaluated across three scenarios. To use this to calculate performance measurements (parameters), the output is copied to the excel sheet. For this simulation, AODV will be evaluated and its performance will be analyzed in terms of average end-to-end delay, average throughput, PDR, total packet failure, and packet size. The simulation parameters and values shall be known for parameters used for simulation in each scenario. As seen in Table 2, which indicates the system environment:

Table 2. Parameters for simulation

\begin{tabular}{|c|c|c|}
\hline No. & Parameters & Specification \\
\hline 1 & MAC Type & $\mathrm{Mac} / 802.11$ \\
\hline 2 & Antenna Model & Omni Antenna \\
\hline 3 & Type of Channel & Wireless Channel \\
\hline 4 & Routing Protocol & AODV, DSDV \& DSR \\
\hline 5 & No. of Nodes & $10,20,30,40$ and 50 \\
\hline 6 & Transmission Protocol & TCP \\
\hline 7 & Interface Queue Type & DropTail/PriQueue \\
\hline 8 & Queue Length & 50 packets \\
\hline 9 & Dimensions $(\mathrm{X}, \mathrm{Y})$ & $653 * 752$ sq.m \\
\hline 10 & Time end for simulation & 300 second \\
\hline 11 & Radio Propagation Model & Propagation /TwoRayGround \\
\hline 12 & Speed & $100 \mathrm{~m} / \mathrm{s}$ \\
\hline 13 & Bandwidth & 2Mbps \\
\hline 14 & Transmission & 2 Ray Ground \\
\hline 15 & Propagation & 2 Ray Ground \\
\hline 16 & Sending Rate & $64 \mathrm{~Kb}$ \\
\hline 17 & Packet Size & 1000 \\
\hline
\end{tabular}

Table 3. Comparison with similar works

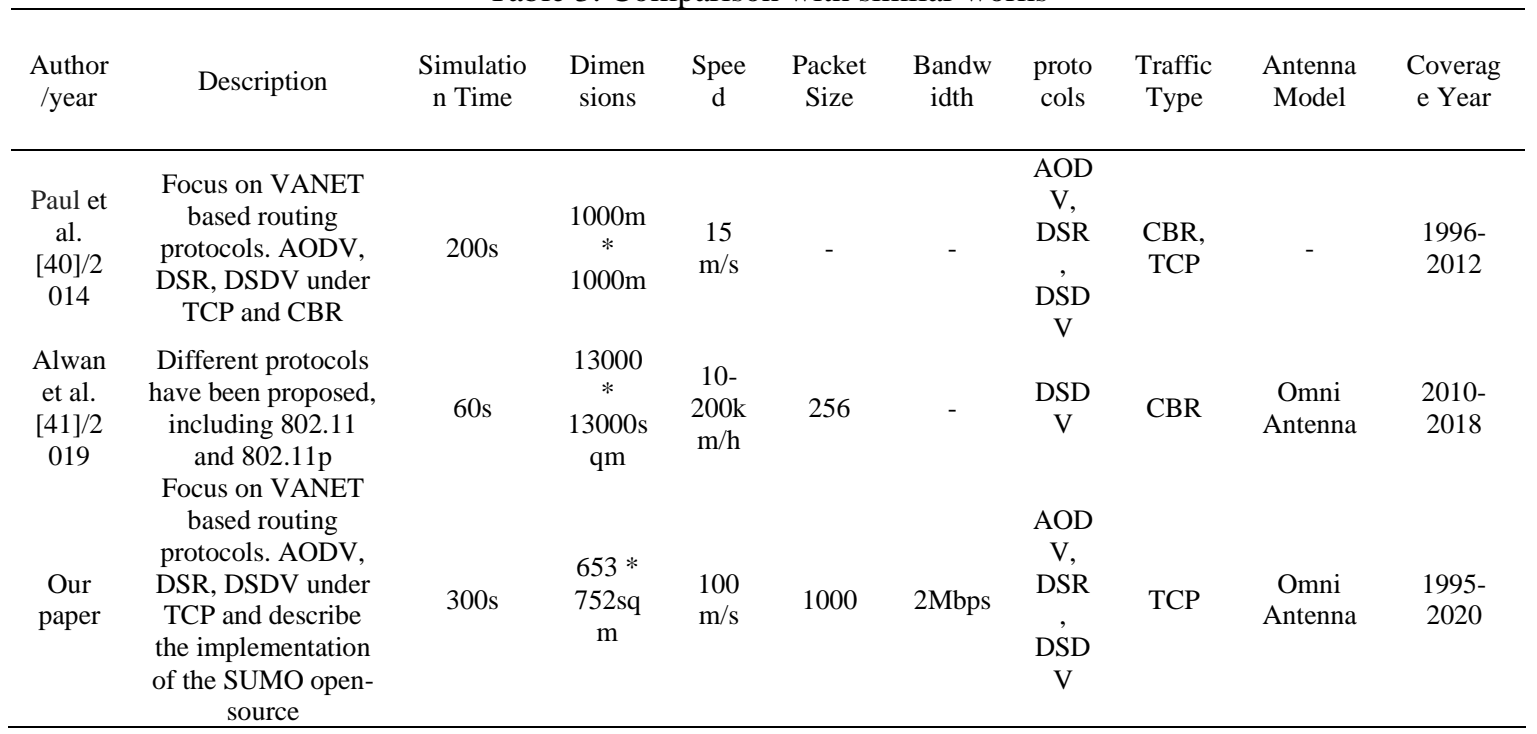

\subsection{NS-2 trace file generator}

As stated earlier, the NS-2 emulation enables one or two trace files to be used because of the simulation. Regular trace file (created with \$ns_trace-all commands) and trace file name (\$ns_name-traceall). A trace file (Nam) is a subset of a normal trace file with the suffix ". nam.". It contains information for the use with the name homonymous tool to visualize the packet flow and node motion. Nam file is a Tcl $\backslash \mathrm{TK}$ animation tool to display network simulation effects and real-world packet tracking data in different network visualization situations [42]. Trace tile is analyzed as already described in this chapter with the following commands, or by excel. An analysis is conducted to predict packet loss, end2end delay, and passage.

\section{RESULTS AND DISCUSSION}

With NS-2 five highway network scenarios developed, each scenario has a different number of nodes that are $(10,20,30,40$, and 50). The specific routing protocol to evaluate is AODV, DSR, and DSDV. 
The evaluation is based on different efficiency measures, then a discussion of the results especially the calculation of traffic impact on the transmission.

\subsection{Simulator setup with five scenarios}

The three common measures used to compare the performance of the above-noted protocols are the performance metrics such as like, throughput, PDR, and E2ED. So, there are five scenarios in this section to test and evaluate the effect of node density and mobility on the performance of VANET routing protocols. Where in the first experiment, the number of nodes (vehicles) is set to 10. And (node 0) is always the source, the destination node will be (node 1) and this applies to all scenarios in this study. The packet transmission shall be set to $10 \mathrm{~ms}$. The first scenario shown in Figure 6 includes 10 nodes. We see that (node 1) shifts to the west and the row indicate that the beams are transmitted directly to the target also inside the source radius. If (node 1) moves forward (node 0), it is out of range (node 1) and packets must travel through the middle node to meet the target.

The second scenario shown in Figure 7 includes 20 nodes. It is planned to transfer (node 0 and node 1) at a faster level, and to link and communicate (node 1) with (node 0). The third scenario shown in Figure 8 includes 30 nodes. Which shows node positions, where node (1) in this scenario in addition to node (0) move more quickly to the far right than the other nodes. And the third scenario is repeated when using (40) nodes in scenario 4 and (50) nodes in scenario 5, as shown in Figures 9 and Figure 10. From the above scenarios, it is obvious that the larger the number of nodes, the more we get a wireless network that operates strongly and appropriately, particularly when using large networks, and this will be discussed in section six.

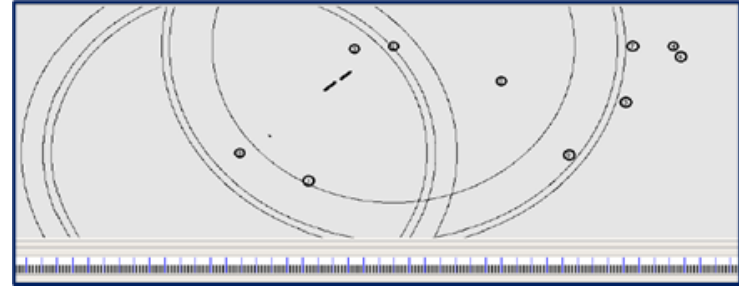

Figure 6. Running a TCL script (scenario 10 nodes)

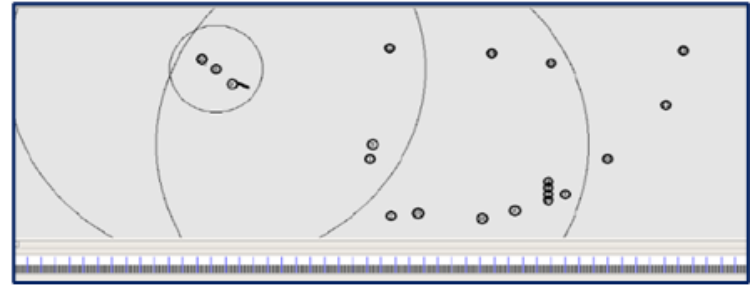

Figure 7. Running a TCL script (scenario 20 nodes)

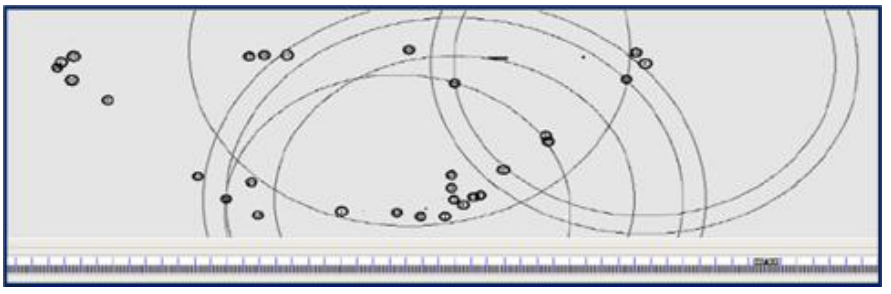

Figure 8. Running a TCL script (scenario 30 nodes)

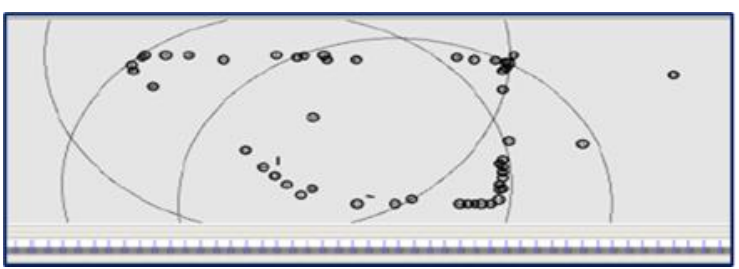

Figure 9. Running a TCL script (scenario 40 nodes)

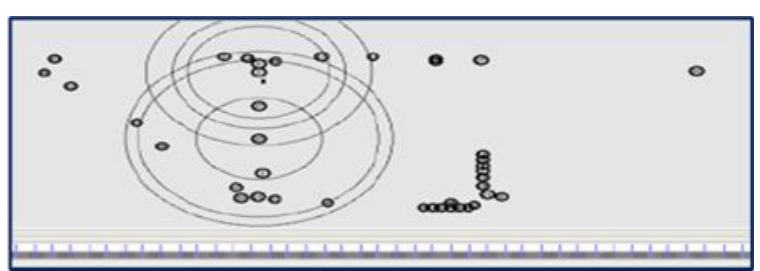

Figure 10. Running a TCL script (scenario 50 nodes)

\subsection{Simulation results and analysis}

\subsubsection{Throughput}

Throughput decreases or drops with increasing nodes between the source and the destination increases. So, when the packet is sent to the third node coming from the second node, the next packet cannot be sent by the first node until more packets are sent to the second node, and so on. Following all routing protocols, I note that the DSR has a better performance compared to the AODV and DSDV protocols as shown in Figure 11. Among the two DSDV and AODV reactive routing protocols, it's found that AODV has 
greater performance than DSDV. DSDV uses on-demand routing and destination sequence numbers, which is the standard method of transfer, while AODV uses source routing, which allows AODV packets to meet their destination in a shorter amount of time and increases their throughput. DSDV has a higher rate of production because when a message enters the destination, the DSDV responds to all requests. Another thing, the node of the target interacts only once with the received requests, while most other requests are ignored. So, it allows AODV to get better throughput than DSDV. When the nodes grow, though, DSR is expected to be even stronger than DSDV and AODV. In Figure 11, we will notice that there is an increase in the number of nodes, and the packet drop decreases, which leads to more throughput.

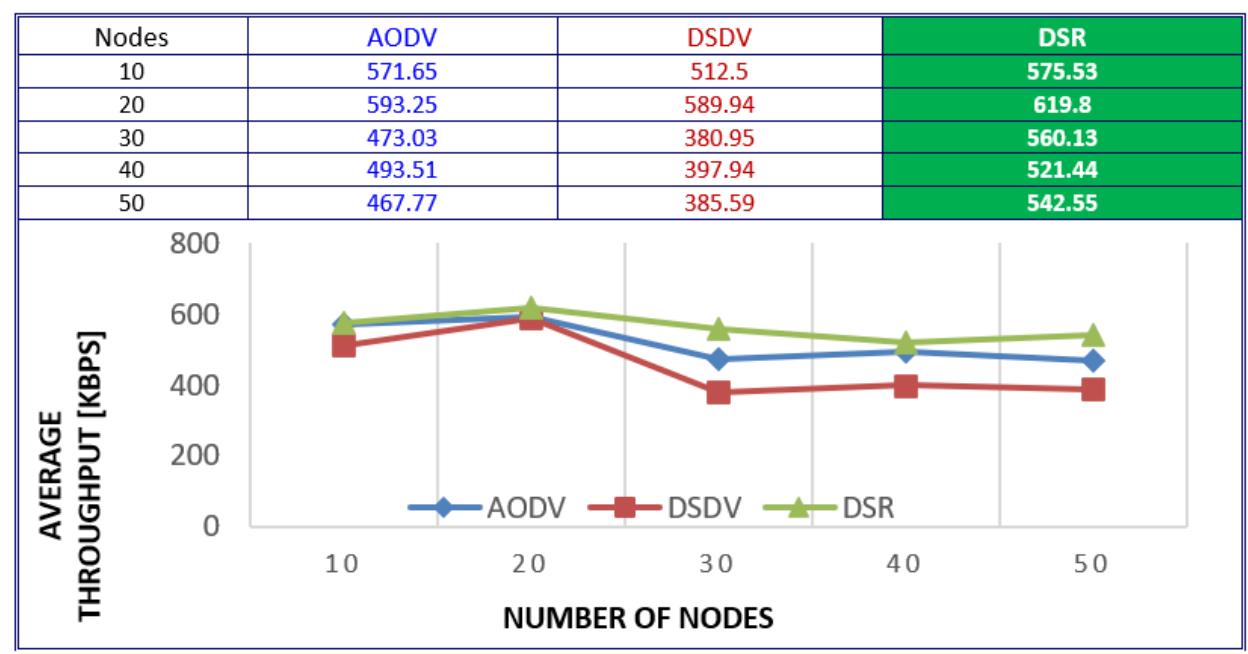

Figure 11. Throughput vs node density

\subsubsection{Packet delivery ratio}

The network performance for this experiment is evaluated based on five different types of simulation [43], as shown in Figure 12. Where (total packet received/total packet send). Figure 12 indicates the ratio of packet transmission to AODV, DSR, and DSDV protocols with five nodes. Notice that particularly at node (30) AODV with fewer nodes has a higher percentage of packet distribution. By comparing AODV and DSDV to DSR, it was found that the DSR had a higher percentage of packet delivery when the node number changed. AODV \& DSDV are interactive routing protocols since the path starts on demand so it needs a long time though to upgrade the path. For what was mentioned, we believe that proactive routing protocols provide a better PDR than others. So DSR is the best among other protocols especially at node (10).

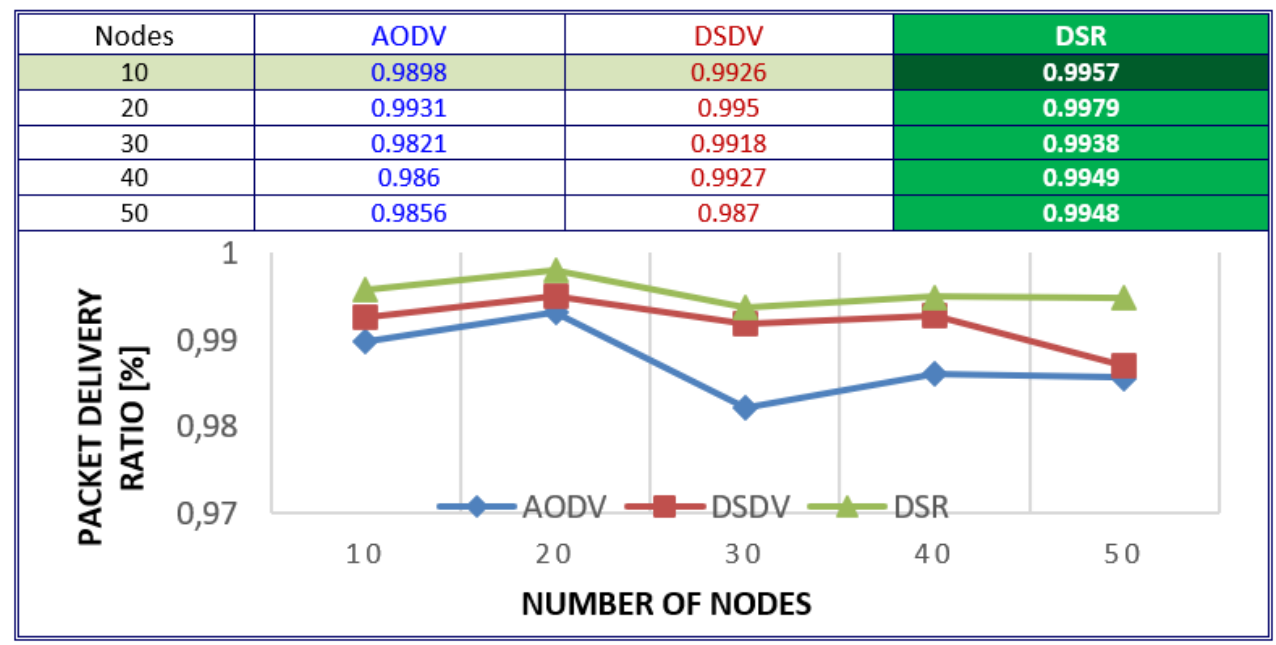

Figure 12. PDR vs node density 


\subsubsection{End-to-end delay}

E2ED for the three chosen VANET with five nodes is seen in this section. E2EDs for the AODV, DSR, and DSDV routing protocols are expressed in milliseconds. The delay in E2ED is tested with the use of five different nodes which are (10-50). E2ED for DSDV is higher from AODV and DSR, especially in node (30). Conservative routing protocols tend to experience with increasing nodes more than responsive routing protocols such as DSDV and thus have more response time from one party to the other. But the DSR protocol has a delay in end-to-end than AODV protocol when comparing the two interactive routing protocols and the explanation is that the DSR requires more time internally to map routes to neighboring boring nodes. Another explanation for the higher DSR lag is that, for comparison with AODV, route detection starts several times. The number of intermediate nodes between the source and the destination increases with the number of nodes increasing. So, it is better to select DSDV in this scenario due to the low end-to-end delay. E2ED vs. node density as shown in Figure 13.

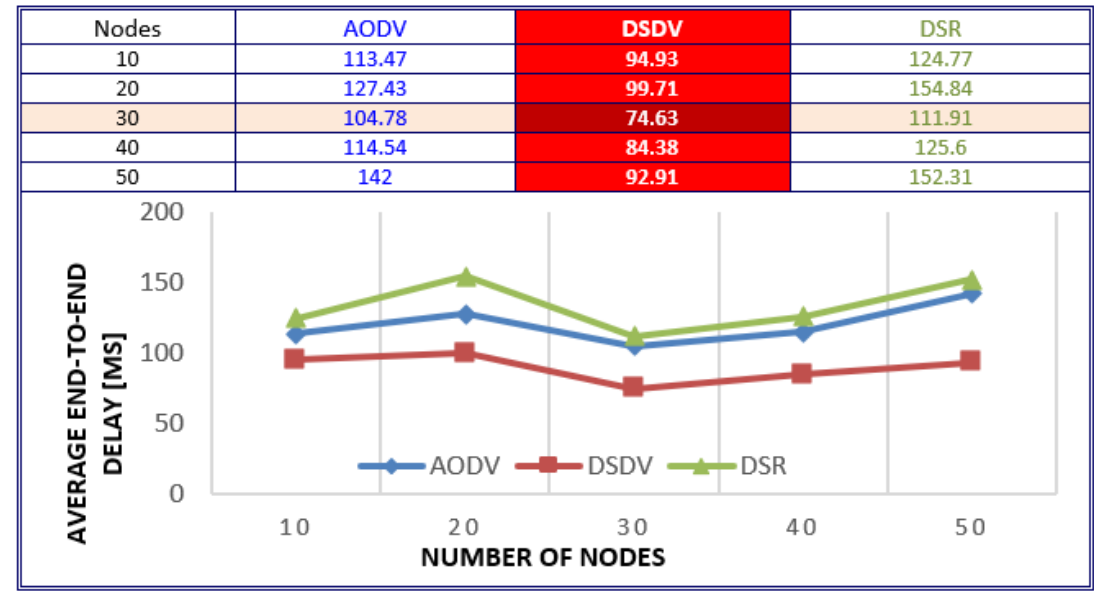

Figure 13. E2ED vs. node density

\subsection{Discussion}

In this paper, the research results are as per the scenarios. Assessment of the routing protocols selected with specific performance measures, where those protocols were measured in terms of (throughput, PDR, E2ED) with three protocols as (AODV, DSDV, and DSR) and we have used five of different nodes. In this paper we were study and density factor of the vehicle's node influencing the performance of routing protocols was analyzed, also the dominance was used to plot graphs to determine performance criterion tests. So, through the results we obtained, it was found that, in terms of efficiency Throughput, DSR performance is much higher than the protocols AODV and DSDV. So, the DSR protocol is the best among other protocols especially at node (10). While, PDR, AODV is too much better than other protocols, especially at node (30). Finally, in E2ED, DSDV is the best choice because of the low average E2ED. This means that any technique or protocol will be suitable from scenario to others, according to the nature of the work required of this protocol to accomplish a specific work.

\section{CONCLUSION}

The two main aim of this paper are, compare between three protocols, and to highlight the simulation process and how to work VANET using available tools in NS-2. It has also been proven that each possible protocol is the best within a specific scenario and at a specific time. Means, the off-line navigation type is created first and then used by a network emulator such as NS-2. AODV, DSDV, and DSR protocols were evaluated to determine the efficacy of VANET routing protocols when node density and mobility change under certain performance metrics, where the vehicle is an important factor in evaluating. As noted in tests, node density has a significant impact on efficiency. The throughput, PDR, E2ED, and overhead costs increase as node density increases. Finally, it can be concluded that these results obtained are largely consistent with the results expected during our theoretical analysis of the protocols. As for three AODV, DSDV, and DSR protocols, it can be concluded the performance of the DSDV protocol was the best in the E2ED, but the DSR protocol was preferable in productivity or throughput and PDR. While, in the PDR, AODV is too much better than other protocols. Consequently, the most popular wireless networking 
applications that work very well are those that can use more nodes. Moreover, each strategy has its own negative and positive aspects that make it ideally suited to a particular scenario than other scenarios. In future work, more nodes use, more analyzes are being done to determine the correct and appropriate routing algorithms for large networks.

\section{REFERENCES}

[1] A. T. Al-Heety, M. T. Islam, A. H. Rashid, H. N. Abd Ali, A. M. Fadil, and F. Arabian, "Performance Evaluation of Wireless data traffic in Mm wave massive MIMO communication," Indonesian Journal of Electrical Engineering and Computer Science (IJEECS), vol. 20, no. 3, pp. 1342-1350, 2020.

[2] P. Manickam, T. G. Baskar, M. Girija, and D. D. Manimegalai, "Performance comparisons of routing protocols in mobile ad hoc networks," arXiv preprint arXiv:1103.0658, 2011.

[3] O. Vermesan and P. Friess, Internet of things: converging technologies for smart environments and integrated ecosystems. River publishers, 2013.

[4] O. A. Hammood et al., "An effective transmit packet coding with trust-based relay nodes in VANETs," Bulletin of Electrical Engineering and Informatics (BEEI), vol. 9, no. 2, pp. 685-697, 2020.

[5] A. S. Mustafa, M. Muthanna Al-Heeti, M. M. Hamdi and A. Muhi Shantaf, "Performance Analyzing the Effect of Network Size on Routing Protocols in MANETs," 2020 International Congress on Human-Computer Interaction, Optimization and Robotic Applications (HORA), Ankara, Turkey, 2020, pp. 1-5.

[6] D. Saha, P. Wararkar, and S. Patil, "Comprehensive Study and Overview of Vehicular Ad-HOC Networks (VANETs) in Current Scenario with Respect to Realistic Vehicular Environment," International Journal of Computer Applications, vol. 975, p. 8887.

[7] A. Dua, N. Kumar, and S. Bawa, "A systematic review on routing protocols for vehicular ad hoc networks," Vehicular Communications, vol. 1, no. 1, pp. 33-52, 2014

[8] M. Hamdi, L. Audah, S. Rashid, A. Mustafa, and M. Abood, "A survey on data dissemination and routing protocol in VANET: Types challenges opportunistic and future role," Int. J. Adv. Sci. Technol, vol. 29, no. 5, pp. 6473-6482, 2020.

[9] C. Raut and S. Devane, "Performance Evaluation of Vehicular Ad Hoc Networks in Case of Road Side Unit Failure," in Cognitive Informatics and Soft Computing: Springer, 2020, pp. 295-305.

[10] S. Ghosal and T. Chatterjee, "Controlling Emergency Vehicles During Road Congestion-A Survey and Solution," in Computational Intelligence in Pattern Recognition: Springer, 2020, pp. 529-538.

[11] A. K. Saluja, S. A. Dargad, and K. Mistry, "A Detailed Analogy of Network Simulators-NS1, NS-2, NS3 and NS4," Int. J. Future Revolut. Comput. Sci. Commun. Eng, vol. 3, pp. 291-295, 2017.

[12] J. Pan and R. Jain, "A survey of network simulation tools: Current status and future developments," Email: jp10@ cse. wustl. edu, vol. 2, no. 4, p. 45, 2008.

[13] T. Issariyakul and E. Hossain, "Introduction to network simulator 2 (NS-2)," in Introduction to network simulator NS-2: Springer, 2009, pp. 1-18.

[14] M. Maad Hamdi, L. Audah, S. Abduljabbar Rashid, A. Hamid Mohammed, S. Alani and A. Shamil Mustafa, "A Review of Applications, Characteristics and Challenges in Vehicular Ad Hoc Networks (VANETs)," 2020 International Congress on Human-Computer Interaction, Optimization and Robotic Applications (HORA), Ankara, Turkey, 2020, pp. 1-7

[15] A. Abuarqoub, F. Al-Fayez, T. Alsboui, M. Hammoudeh, and A. Nisbet, "Simulation issues in wireless sensor networks: A survey," in The Sixth International Conference on Sensor Technologies and Applications (SENSORCOMM 2012), 2012, pp. 222-228.

[16] F. Tabbana, "Performance Analysis of AODV, DSDV and ZRP Routing Protocols for Wireless Sensor Networks using NS-2 Tool," vol. 10, no. 5, pp. 279-297, 2020.

[17] S. A. Rashid, L. Audah, M. M. Hamdi, M. S. Abood, and S. Alani, "Reliable and efficient data dissemination scheme in VANET: a review," International Journal of Electrical and Computer Engineering (IJECE), vol. 10, no. 6, pp. 6423-6434, 2020

[18] M. H. Alwan, K. N. Ramli, A. Z. Ameer Alhasan, and H. F. Sameen, "Qos-aware SNR Admission Control Mechanism," Journal of Theoretical and Applied Information Technology, vol. 97, no. 3, 2019.

[19] G. Khanna and S. K. Chaturvedi, "A comprehensive survey on multi-hop wireless networks: milestones, changing trends and concomitant challenges," Wireless Personal Communications, vol. 101, no. 2, pp. 677-722, 2018.

[20] A. Rahim et al., "Vehicular social networks: A survey," Pervasive and Mobile Computing, vol. 43, pp. 96-113, 2018.

[21] R. Pandit and V. Richariya, "Performance Evaluation of Routing Protocols for Manet using NS-2," International Journal of Computer Applications, vol. 66, no. 24, 2013.

[22] A. K. Basil, M. Ismail, M. A. Altahrawi, H. Mahdi and N. Ramli, "Performance of AODV and OLSR routing protocols in VANET under various traffic scenarios," 2017 IEEE 13th Malaysia International Conference on Communications (MICC), Johor Bahru, Malaysia, 2017, pp. 107-112.

[23] M. M. Shah, S. Serasia, and H. Patel, "Simulation-based performance evaluation of routing protocols for parking," International Journal of Computer Science and Mobile Computing, vol. 4, no. 4, pp. 335-340, 2015.

[24] L. Rivoirard, M. Wahl, P. Sondi, M. Berbineau and D. Gruyer, "Performance evaluation of AODV, DSR, GRP and OLSR for VANET with real-world trajectories," 2017 15th International Conference on ITS Telecommunications (ITST), Warsaw, Poland, 2017, pp. 1-7. 
[25] P. A. Lopez et al., "Microscopic Traffic Simulation using SUMO," 2018 21st International Conference on Intelligent Transportation Systems (ITSC), Maui, HI, USA, 2018, pp. 2575-2582.

[26] S. Boussoufa-Lahlah, F. Semchedine, and L. Bouallouche-Medjkoune, "Geographic routing protocols for Vehicular Ad hoc NETworks (VANETs): A survey," Vehicular Communications, vol. 11, pp. 20-31, 2018.

[27] C. Tripp-Barba, A. Zaldívar-Colado, L. Urquiza-Aguiar, and J. A. Aguilar-Calderón, "Survey on Routing Protocols for Vehicular Ad Hoc Networks Based on Multimetrics," Electronics, vol. 8, no. 10, p. 1177, 2019.

[28] D. Soumaya, S. Dardouri, and B. Ridha, "Performance Evaluation of Routing Protocols for Vehicular AD-HOC Networks Using NS-2/SUMO," in Workshops of the International Conference on Advanced Information Networking and Applications, 2020, pp. 352-365: Springer.

[29] P. Rani, N. Sharma and P. K. Singh, "Performance Comparison of VANET Routing Protocols," 2011 7th International Conference on Wireless Communications, Networking and Mobile Computing, Wuhan, China, 2011, pp. 1-4.

[30] P. K. Singh, K. Lego, and T. Tuithung, "Simulation based analysis of adhoc routing protocol in urban and highway scenario of VANET," International Journal of Computer Applications, vol. 12, no. 10, pp. 42-49, 2011.

[31] B. Makodia, T. Patel, K. Parmar, S. K. Hadia and A. M. Shah, "Implementing and analyzing routing protocols for self-organized vehicular adhoc network," 2013 Nirma University International Conference on Engineering (NUiCONE), Ahmedabad, India, 2013, pp. 1-6.

[32] S. Daud, S. M. M. Gilani, M. S. Riaz and A. Kabir, "DSDV and AODV Protocols Performance in Internet of Things Environment," 2019 IEEE 11th International Conference on Communication Software and Networks (ICCSN), Chongqing, China, 2019, pp. 466-470.

[33] M. K. Hasan and O. Sarker, "Routing protocol selection for intelligent transport system (ITS) of VANET in high mobility areas of Bangladesh," in Proceedings of International Joint Conference on Computational Intelligence, 2020, pp. 123-135: Springer.

[34] P. K. Singhal and V. Chaubey, "An Enhanced Ad Hoc on Demand Distance Vector Routing Protocol for Vehicular Ad Hoc Networks (VANET's)," in Proceedings of International Conference on Sustainable Computing in Science, Technology and Management (SUSCOM), Amity University Rajasthan, Jaipur-India, 2019.

[35] S. Malik and P. K. Sahu, "A comparative study on routing protocols for VANETs," Heliyon, vol. 5, no. 8, p. e02340, 2019.

[36] F. K. Karnadi, Z. H. Mo and K. Lan, "Rapid Generation of Realistic Mobility Models for VANET," 2007 IEEE Wireless Communications and Networking Conference, Hong Kong, China, 2007, pp. 2506-2511.

[37] T. Kaur and A. Verma, "Simulation and Analysis of AODV routing protocol in VANETs," International Journal of Soft Computing and Engineering (IJSCE), vol. 2, no. 3, pp. 2231-2307, 2012.

[38] I. Khan, "Performance evaluation of Ad hoc routing protocols for Vehicular ad hoc networks," Mohammad Ali Jinnah University, 2009.

[39] Z. Fu, "GeoSparkSim: A Scalable Microscopic Road Network Traffic Simulator Based on Apache Spark," Arizona State University, 2019.

[40] B. Paul, A. Roy and S. K. Paul, "Comparison of DSR, AODV, and DSDV Routing Protocols with Varying Pause Time \& Node Density over TCP \& CBR Connections in VANET," 2014 International Conference on Computational Intelligence and Communication Networks, Bhopal, India, 2014.

[41] M. H. Alwan, K. N. Ramli, Y. A. Al-Jawher, A. Z. Sameen, and H. F. Mahdi, "Performance comparison between 802.11 and $802.11 \mathrm{p}$ for high speed vehicle in VANET," International Journal of Electrical and Computer Engineering, vol. 9, no. 5, p. 3687, 2019.

[42] J. Plehn, "The design of location areas in a GSM-network," 1995 IEEE 45th Vehicular Technology Conference. Countdown to the Wireless Twenty-First Century, Chicago, IL, USA, 1995, pp. 871-875 vol.2.

[43] S. A. Yah, N. Yaakob, M. E. Elobaid, O. B. Lynn, R. Badlishah, and W. A. N. W. Abdullah, "Newton-raphson method to solve systems of non-linear equations in VANET performance optimization," Bulletin of Electrical Engineering and Informatics, vol. 8, no. 1, pp. 336-342, 2019. 